Clemson University

TigerPrints

Publications

Holcombe Department of Electrical \& Computer

Engineering

2019

(Invited) Emerging Role of Silicon Carbide and Gallium Nitride Based Power Electronics in Power and Transportation Sectors

Prahaladh Paniyil

Rajendra Singh

Follow this and additional works at: https://tigerprints.clemson.edu/elec_comp_pubs

Part of the Automotive Engineering Commons, and the Electrical and Computer Engineering Commons 


\section{(Invited) Emerging Role of Silicon Carbide and Gallium Nitride Based Power Electronics in Power and Transportation Sectors}

To cite this article: Prahaladh Paniyil and Rajendra Singh 2019 ECS Trans. 923

View the article online for updates and enhancements. 


\title{
Emerging Role of Silicon Carbide and Gallium Nitride Based Power Electronics in Power and Transportation Sectors
}

\author{
Prahaladh Paniyil ${ }^{\mathrm{a}}$, Rajendra Singh ${ }^{\mathrm{a}, \mathrm{b}}$ \\ ${ }^{\text {a }}$ Holcombe Department of Electrical and Computer Engineering, Clemson University, \\ Clemson, South Carolina 29634, USA \\ ${ }^{b}$ Department of Automotive Engineering, Clemson University, Clemson, SC 29607 USA
}

Free fuel-based energy sources (solar and wind) are pointing towards a future in which sustainable energy is affordable, abundant and deployed with high energy efficiency. Driven by advancements in the technology of lithium batteries and the availability of low-cost sustainable clean electric power, the electrification of transportation is going through fundamental disruptive transformation. Without any doubt, both power and transportation sectors will provide phenomenal growth of power electronics in the $21^{\text {st }}$ century. Recently, both $\mathrm{SiC}$ and $\mathrm{GaN}$ are drawing the attention as potential replacement of Si-based power electronics. These may open some new markets where Si based power electronics cannot function either due to power or temperature limitations. In this paper we have identified the WBG based key power electronics products that should be focused to see their high growth.

\section{Introduction}

Similar to low-power electronics, silicon is also playing a dominant role in power electronics. The last two decades of progress of free fuel-based energy sources namely solar and wind as well as electrification of transportation is providing fundamental disruptive transformations where silicon cannot meet the performance requirement of future power electronics in the energy and transportation sectors. These new opportunities are driven by advancements in the technology of lithium batteries (cost reduction and increase in energy density). Volume manufacturing, similar to photovoltaic modules is mainly responsible for the cost reduction of lithium ion batteries (1). Wide Bandgap (WBG) materials namely silicon carbide ( $\mathrm{SiC})$ and gallium nitride $(\mathrm{GaN})$ have the potential to meet the performance requirements of future power electronics in the energy and transportation sectors. Although there has been limited success in the implantation of power electronics based on $\mathrm{SiC}$ and $\mathrm{GaN}$, cost reduction on global scale has been the main problem. The objective of this paper is to provide the emerging role of WBG based power electronics and the challenge of cost reduction.

\section{Potential for WBG Electronics}

Recently, there has been an increase in the incorporation of more efficient and reliable systems in all major industrial sectors. The usage of Direct Current (DC) power has substantially increased over the last few decades. As an example, data centers are now implementing a $380 \mathrm{~V}$ DC power networks. The DC power network has enabled this sector to eliminate power conversion losses and increase overall efficiency. The reduction in the 
number of components due to this new architecture has also substantially reduced component cost, area and weight for the data center systems. The existing power distribution network is still largely based on AC transmission and distribution networks. Due to this shift from Alternating Current (AC) networks to DC networks, there is a need for new and efficient power inverting systems. Also, the conversion of voltage levels in a DC network is not as straightforward as an AC transformer. A transformer can easily stepup or step-down AC voltage levels. DC networks on the other hand need DC-DC converters that are possible today due to the emerging power transistors. Switching techniques have already established their superiority over other DC-DC conversion techniques. Switching can be most effectively carried out by power transistors. Thus, the increasing demand for DC-DC conversion and AC-DC inversion can be directly corelated to the demand for power transistors. The possible applications include wind energy, solar PV, Motor drives, Electronic Appliances, power supplies, UPS, Internet-Of-Things, servers and data centers, railways and electric vehicles (EV). The sales history and projections for power modules according to applications are given in (2) and indicates the demand for power electronics in the modern era.

The traditional Si MOSFETs have provided effective low voltage $(<600 \mathrm{~V})$ switching circuits for internal DC voltage conversion in various circuits. However, their performance struggles above 600V. A new class of Si MOSFETs called the Si Super-Junction (SJ) MOSFETs have also attracted a lot of attention lately. These new MOSFETs have a new device structure and is claimed to handle voltages much higher than most Si Power MOSFETs. SJ MOSFETs have a larger area of $\mathrm{p}-\mathrm{n}$ junction enabling a higher breakdown voltage. However, the SJ MOSFETs also have limited capabilities at higher operating voltages and power and requires a considerable amount of research and development. Thus, the introduction of Si Insulated Gate Bipolar Transistor (IGBT) is crucial to our context. The IGBT combines the characteristics of the Bipolar Junction Transistor (BJT) and MOSFET. BJTs have better output performance than a MOSFET in terms of handling higher currents while the MOSFET is voltage-controlled rather than current-controlled. Thus, the MOSFET can provide a much higher frequency of switching than BJTs but BJTs can operate at higher power outputs. The IGBT thus combines the high-power output characteristic of the BJT with the precision of voltage-controlled input of the MOSFET. The IGBTs operate at a lower switching frequency than a power MOSFET but can handle much higher voltages and output power. Due to the extensive demand, there was a need to switch to other technologies and materials to expand the transistor functionalities for higher ratings. The material properties of WBG materials catered to these requirements. $\mathrm{SiC}$ and $\mathrm{GaN}$ are the major materials in the transistor sector today and will be our focus.

\section{Comparison of Power Characteristics for Si, SiC and GaN Power Transistors}

The higher bandgap of $\mathrm{SiC}$ and $\mathrm{GaN}$ transistors enable various characteristics over $\mathrm{Si}$ transistors. These characteristics are associated with switching speed, input and output impedance, voltage and current, temperature coefficients and power losses. Firstly, power losses with respect to switching frequency at $150^{\circ} \mathrm{C}$ is shown in Fig. 1. The data to populate the graph is obtained from (3). It can be clearly deduced from the graph that GaN FETs have much better output power at high temperature and higher switching frequencies. 


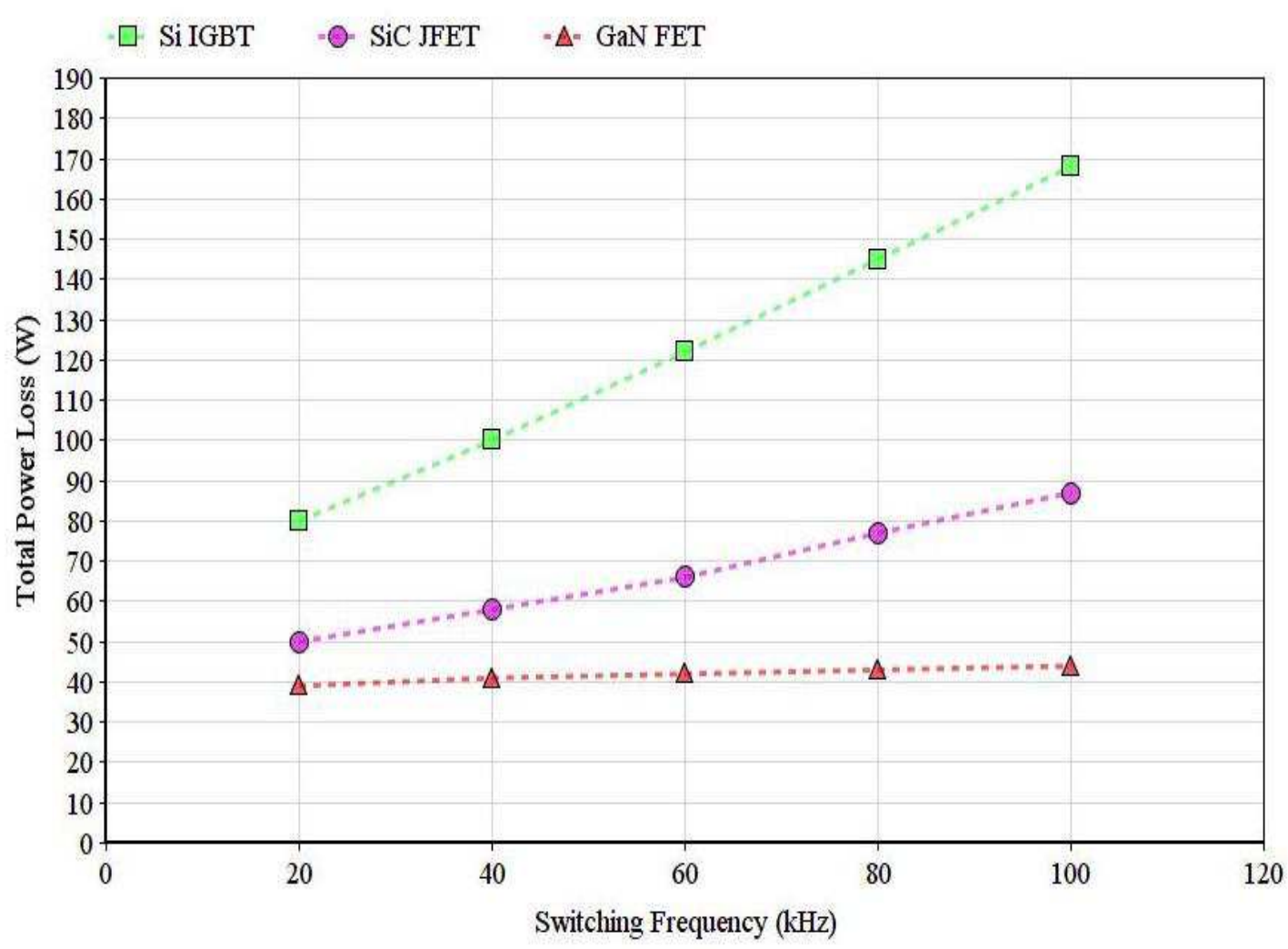

Figure 1. Power loss vs Switching Frequency for Transistors at $150^{\circ} \mathrm{C}$

A more detailed study on the temperature characteristics with respect to bandgap, carrier mobility, saturation velocity, thermal conductivity and converter efficiencies can be found in (3). The bottom-line analysis from (3) is that GaN FETs have much better temperature and efficiency characteristics compared to Si IGBT and SiC JFET except for thermal conductivity. The thermal conductivity for $\mathrm{SiC}$ is superior to $\mathrm{GaN}$ due to its material properties. Due to its limited thermal conductivity, GaN FETs are stagnated at voltage levels of about 600-650V. However, new design considerations for GaN FETs are being researched extensively to implement these transistors in the higher voltage range of $>600 \mathrm{~V}$.

The $\mathrm{SiC}$ transistors can operate above $650 \mathrm{~V}$ due to their high thermal conductivity but the switching frequency is considerably less as compared to $\mathrm{GaN}$ transistors. SiC transistors are stated to operate at considerably high voltage levels of even $>1200 \mathrm{~V}$. At such high voltage levels, $\mathrm{SiC}$ transistors do not face a competition from $\mathrm{GaN}$ transistors. $\mathrm{Si}$ IGBTs, although operating at a lower frequency than $\mathrm{SiC}$ or $\mathrm{GaN}$, shows good performance characteristics at such high voltage levels. Si IGBTs also have a great cost advantage which will be discussed in the following sections of this paper. Our research into operating voltage and frequency regions for various transistors can be cumulated into Fig. 2. 


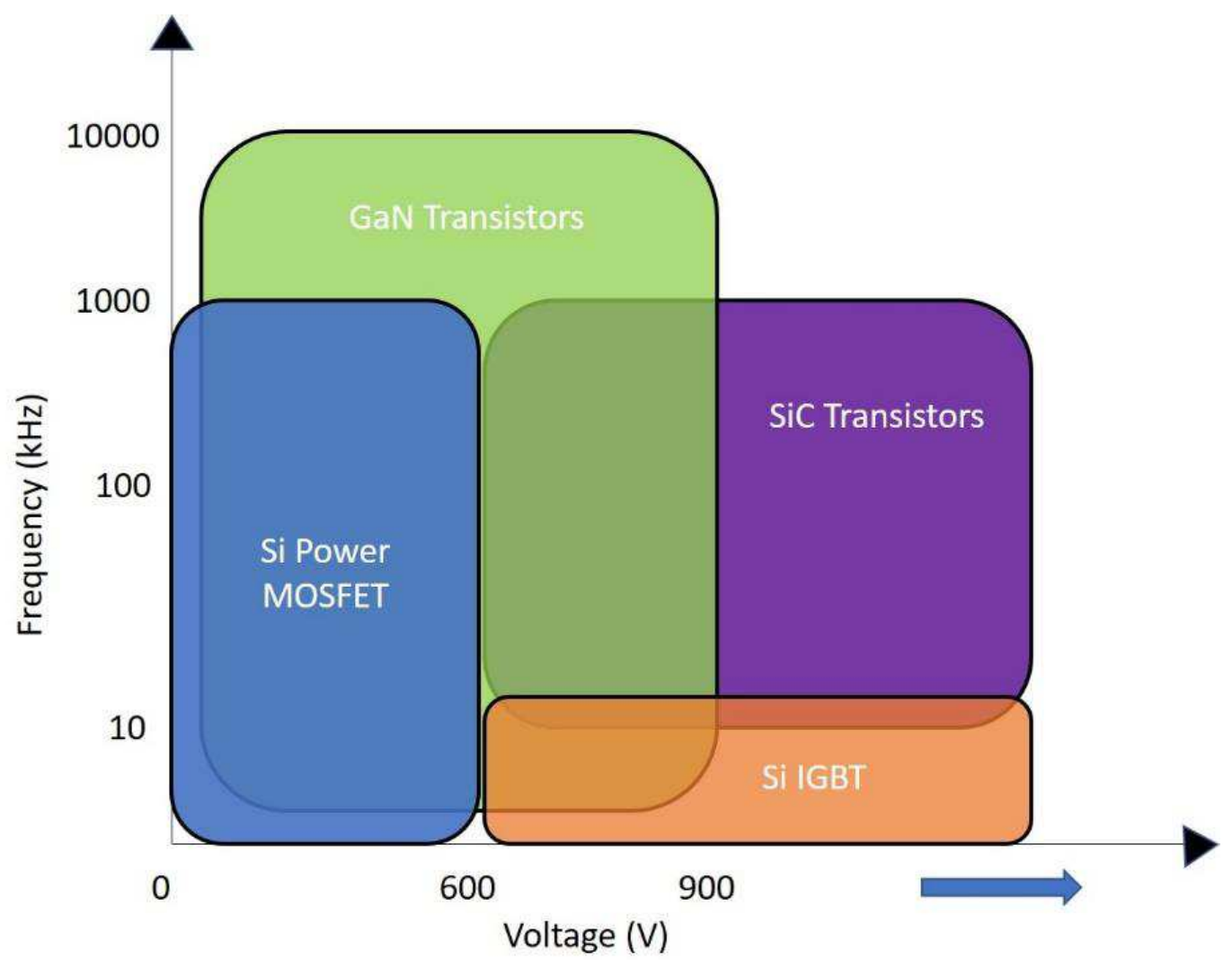

Figure 2. Approximate transistor operating classification

The areas shown in this figure only give an approximation of the transistor characteristics and many manufacturers claim to have engineered a variety of transistors to surpass these limitations. However, in lieu of our discussion Fig. 2 gives out a decent approximation for the different types of transistors and their desired operating voltage and frequencies. The power transistors utilized in the energy and transportation sector constitute the overlapping application region between $600-900 \mathrm{~V}$. The three major material transistor types must cater to the needs of these sectors while keeping up with the demand at the most appropriate cost to market. The argument in this paper is focused on discussing the viability of WBG power electronics in these sectors. Various factors like area, power density, component and system weight, switching frequencies, scaling, etc. contribute to the total system cost in power electronics. Although WBG power electronics systems are priced higher than their Si counterparts, these factors impact their feasibility in the fastgrowing energy and transportation sectors. Firstly, we will analyze power electronics in the energy sector.

\section{Role of Power Transistors in the Sustainable Energy Sector}

The power sector is currently undergoing a disruptive change owing to the need for a low-cost, cleaner, more efficient, more reliable and sustainable energy source. Both wind and photovoltaics (PV) have shown substantial growth over the years. Table I (4) shows faster growth of PV over wind in future We have emphasized the growth of solar PV systems in our recent research publication (5). 
TABLE I. Past and Near Future Growth of PV and Wind

Energy based installed systems (4).

\begin{tabular}{ccccc}
\hline $\begin{array}{c}\text { Wind and Solar } \\
\text { (worldwide) }\end{array}$ & $\begin{array}{c}\text { Wind Total } \\
\text { Capacity }\end{array}$ & $\begin{array}{c}\text { Wind New } \\
\text { Installations }\end{array}$ & $\begin{array}{c}\text { Solar Total } \\
\text { Capacity }\end{array}$ & $\begin{array}{c}\text { Solar New } \\
\text { Installations }\end{array}$ \\
\hline 2019 Forecast & $655.9 \mathrm{GW}$ & $65.4 \mathrm{GW}$ & $646.8 \mathrm{GW}$ & $137.5 \mathrm{GW}$ \\
2018 Preliminary & $591.5 \mathrm{GW}$ & $51.3 \mathrm{GW}$ & $509.3 \mathrm{GW}$ & $102.4 \mathrm{GW}$ \\
$\begin{array}{c}2017-2018 \\
\text { Change }\end{array}$ & $+10.9 \%$ & $+27.4 \%$ & $+27.0 \%$ & $+34.3 \%$ \\
\hline
\end{tabular}

PV produces a DC output which is then boosted to another voltage level to convert to $\mathrm{AC}$ for utilization as the mains supply voltage. Wind turbines produce AC but nowadays many turbines first convert their output to DC in order to achieve the maximum power output from varying wind speeds. Thus, DC to AC power inverters are crucial in accommodating a power network based on renewable energy. The high power, switching transistors are effective in converting (inverting) the DC output from PV and wind turbines to an AC output that can be utilized by the power transmission and distribution network. The role of power transistors in the energy sector can be extrapolated by the investigation of a PV-based power system. A typical inversion block schematic from a 36V PV system to $230 \mathrm{~V}-50 \mathrm{~Hz}$ AC output using Maximum Power Point Tracking (MPPT) is shown in Fig. $3(6)$.

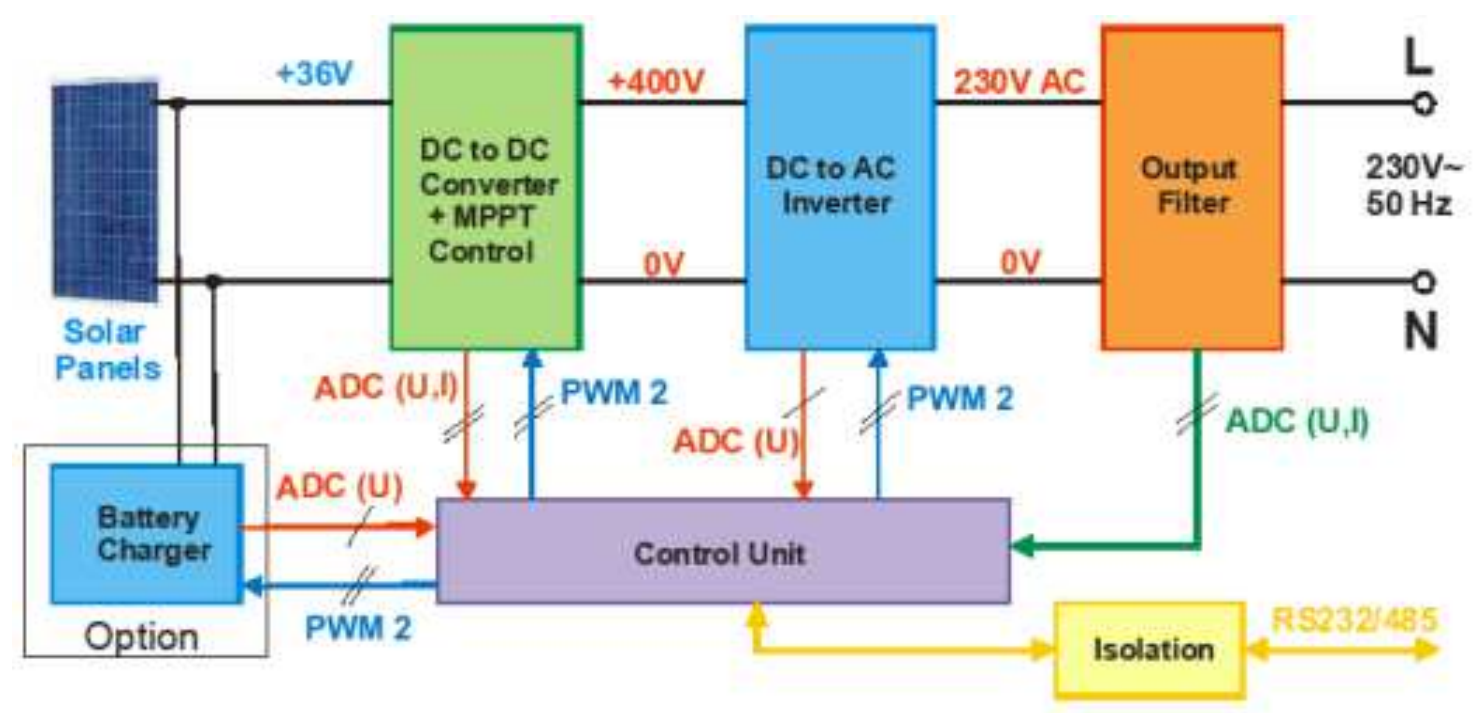

Figure 3. PV Power System using MPPT inversion for AC output (6)

The DC to DC Converter utilizes high frequency switching transistors to boost the DC voltage level to $400 \mathrm{~V}$. This high voltage DC output serves as the input for the DC to AC inverter which inverts the DC voltage to the desired AC voltage by implementing a transistor inverter topology. Finally, an LC filter circuit drives the inverter output to the desired frequency for the $230 \mathrm{~V}$ AC output. In this schematic, the PWM microcontrollers serve as the control logic for the MPPT algorithm for maximum output efficiency from the solar panels. Also, the battery charging circuit utilizes the power generated by PV for charging a battery to supply constant power during the unavailability of solar power at night. Power electronics, thus, is the pivot of this system contributing to the DC voltage boost, inversion and battery charging circuit. 
DC voltage conversion can be implemented using various topologies as seen in (7). The boost converter is the most relevant topology in converting to such high DC voltage levels. We can consider a push-pull topology for DC-DC boost as mentioned in (6). Fig. 4 (6) indicates the power transistors being utilized in the DC-DC boost converter. The input voltage from the PV is switched by the transistors at a high frequency to give a pulsed DC output to the transformer. The transformer steps up the voltage level depoending on its turns ratio to provide a high DC voltage level in pulsed form to be converted to a stable DC voltage $(400 \mathrm{~V})$ by the diodes and LC tank circuit.

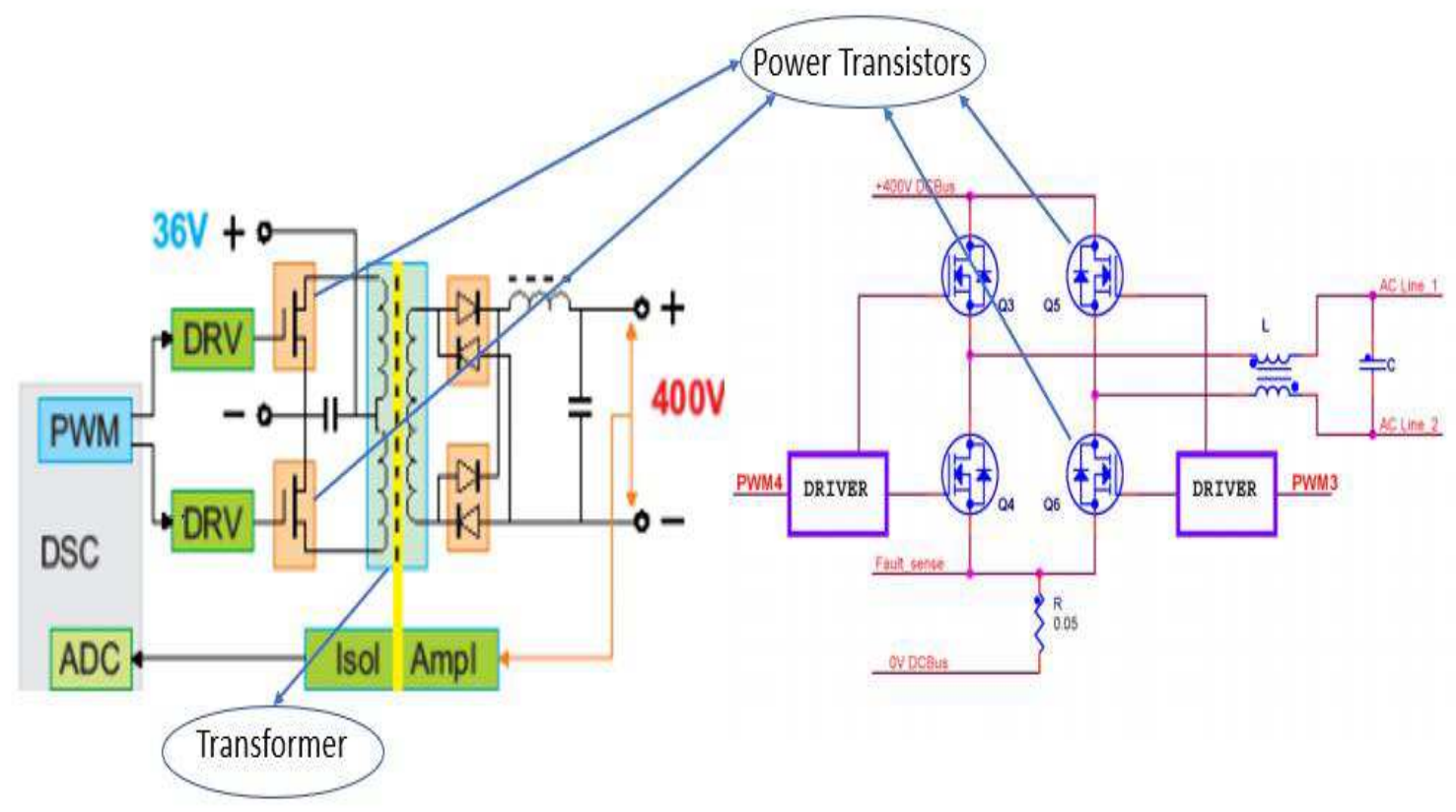

Figure 4. DC-DC Boost converter for $36 \mathrm{~V}$ to $400 \mathrm{~V}$ Conversion and DC-AC full bridge inverter for $400 \mathrm{Vdc}$ to $230 \mathrm{Vac}$ Inversion (6)

The 400V DC output from the boost converter is the input for the inverter circuit. There are various configurations for inversion mechanisms using power transistors. The configuration illustrated in Fig. 4 is the full bridge configuration. This configuration is similar to a full wave rectifier but converts the DC voltage to pulsating DC instead of converting from $\mathrm{AC}$ to $\mathrm{DC}$. The pulsating $\mathrm{DC}$ is then converted using a transformer to the desired $\mathrm{AC}$ voltage. The switching frequency of the power transistors directly corelates to efficieny of boosting and inverting by the transformer. However, the transistors suffer switching losses at higher frequencies. Fig. 5 (8) shows the simulated power losses with respect to increasing frequencies. GaN FETs have better performance at higher frequencies than Si Power MOSFETs or SiC FETs. Also, as seen in the previous section, the temperature performance of $\mathrm{SiC}$ is superior to $\mathrm{Si}$ or GaN FETs. At high frequency operation, there is considerable heat discharge from the transistors. Thus, it is necessary to facilitate heat sinks in the circuit which increase the size and weight of the system. Since a high frequency operation is considered in this case, Si Power MOSFETs (Si SJ MOSFET) is of greater emphasis than the Si IGBT. 


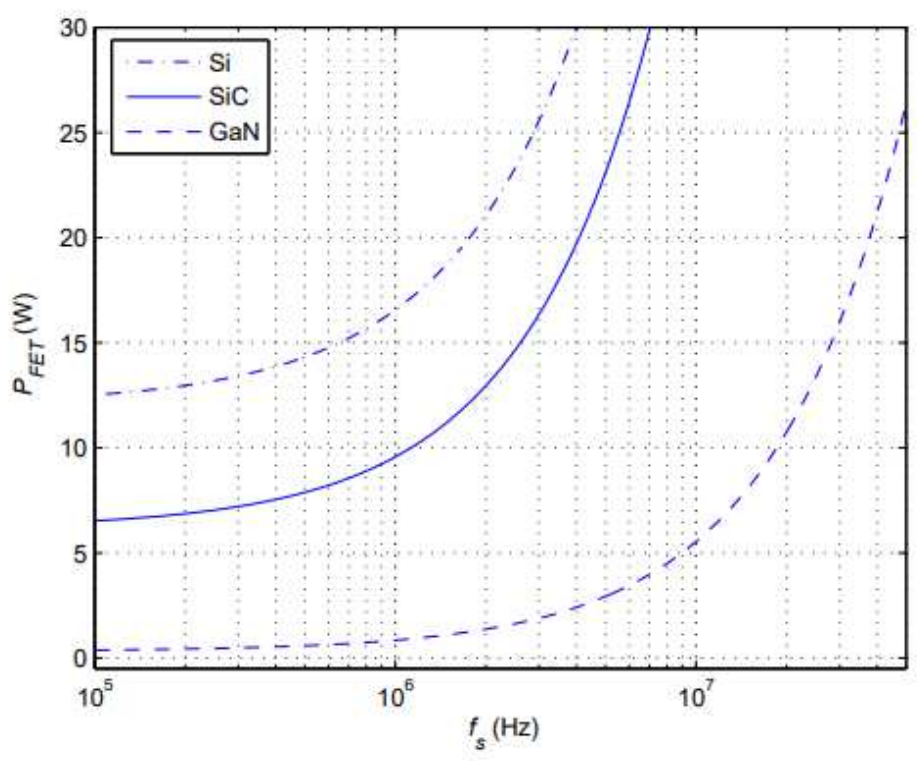

Figure 5. Simulated power losses for $\mathrm{Si}, \mathrm{SiC}$ and $\mathrm{GaN}$ transistors with respect to increasing switching frequency (8)

Another block of interest in Fig. 4 is the DRV block which indicates the gate drivers for the transistors. Si MOSFETs and GaN FETs both utilize symmetric rails and hence have straightforward gate drives which do not significantly contribute to the system cost. $\mathrm{SiC}$ transistors have an asymmetric rail and require a small negative voltage to ensure their OFF state (9). The gate driver requirement is an additional system component to $\mathrm{SiC}$ systems. The three factors of switching loss, heat dissipation and gate drivers contribute to the system cost for this particular system and must be taken into consideration while choosing the best transistor for implementation. The power requirements for inveters depends on the application perspective for solar inverters. Residential, commercial and utility-scale solar PV installation need higher power handling capabilities in increasing order respectively. The common types of inverters for different power scales are micro (low power range), string inverters (medium power range) and central inverters (high power range) (10). Selecting the best inverter configuration for different scales of PV application is altogether another area of research and is not in our scope of discussion for this paper. However, selecting switching transistors with cost effectiveness for our application is of relevance. Let us first compare transistors for the DC-DC conversion stage. The DC-DC conversion stage can operate on voltage levels as low as $200 \mathrm{~V}$ and can go up to more than $1 \mathrm{kV}$ depending on the type of inverter (10). For voltages below $650 \mathrm{~V}$ the real choice is between Si MOSFETs and GaN FETs. The switching frequencies for both these transitors are considerably high for these voltage levels. However, Si MOSFETs are limited by their power dissipation which cannot match that of GaN FETs. The Si MOSFETs have lesser power disaipation than GaN FETs and thus result in much larger systems due to the inclusion of heat sinks. GaN FETs dissipate much more power at the same switching frequency and are an attractive choice for smaller systems. We have generated a comparison table (Table II) based on search results for a $650 \mathrm{~V}$ and $20 \mathrm{~A}$ power transistor from Mouser (11). These transistors are from different manufacturers for different technologies for our specifications. The selection of these specific transistors is to justify our argument. A variety of new transistors based on new technologies are emerging everyday. 
TABLE II. Comparison of transistor technologies for 650V/20A requirement. (12)(13)(14)(15)(16)(17)

\begin{tabular}{cccc}
\hline Manufacturer & Technology & Power dissipation & Cost per transistor \\
\hline ON Semiconductor & Si SuperJunction & $39 \mathrm{~W}$ & $\$ 2.88$ \\
Vishay & Si MOSFET & $33 \mathrm{~W}$ & $\$ 3.64$ \\
STMicroelectronics & Si MDMesh & $30 \mathrm{~W}$ & $\$ 2.91$ \\
Transphorm & GaN on Si & $96 \mathrm{~W}$ & $\$ 11.00$ \\
ROHM Semiconductor & $\mathrm{SiC}$ & $103 \mathrm{~W}$ & $\$ 11.94$ \\
Infineon & Si CoolMOS & $128 \mathrm{~W}$ & $\$ 5.64$ \\
\hline
\end{tabular}

From Table II, the power dissipation for the last 3 transistors are quite attractive. The cost difference between the GaN and SiC transistors with the Si Power MOSFETs is also illustrated in the table. The Infineon MOSFET is a different technology than the other Si MOSFETs and classifies as a higher class of SJ MOSFET. There are other SJ MOSFETs that have emerged recently with similar costs and characteristics. Also, the negative gate drive for $\mathrm{SiC}$ transistor decreases its viability in the competition due to the availability of $\mathrm{GaN}$ and Si SJ transistors at better costs. Thus, the competition for the DC-DC conversion stage is between GaN and Si SJ transistors. Unless GaN transistors prove their feasibility in the power dissipation or cost department, Si transistors seem to be winning this battle.

Now, the DC-AC conversion stage requires transistors to operate at voltages higher than $650 \mathrm{~V}$. As in the DC-DC conversion stage, we can compare transistors for our deisred voltage and current level for different technologies.

TABLE III. Comparison of transistor technologies for $>650 \mathrm{~V} / 20-30 \mathrm{~A}$ requirement. $(18)(19)(20)(21)(22)(23)$

\begin{tabular}{ccccc}
\hline Manufacturer & Technology & $\begin{array}{c}\text { Voltage and } \\
\text { Current }\end{array}$ & $\begin{array}{c}\text { Power } \\
\text { dissipation }\end{array}$ & $\begin{array}{c}\text { Cost per } \\
\text { transistor }\end{array}$ \\
\hline STMicroelectronics & Si MDMesh & $900 \mathrm{~V} / 20 \mathrm{~A}$ & $250 \mathrm{~W}$ & $\$ 6.33$ \\
ON Semiconductor & Si SuperJunction & $800 \mathrm{~V} / 23 \mathrm{~A}$ & $278 \mathrm{~W}$ & $\$ 3.48$ \\
Cree, Wolfspeed & SiC FET & $900 \mathrm{~V} / 23 \mathrm{~A}$ & $97 \mathrm{~W}$ & $\$ 6.43$ \\
Infineon & Si IGBT & $1.2 \mathrm{kV} / 20 \mathrm{~A}$ & $100 \mathrm{~W}$ & $\$ 6.56$ \\
$\begin{array}{c}\text { ROHM } \\
\text { Semiconductor }\end{array}$ & SiC FET & $1.2 \mathrm{kV} / 31 \mathrm{~A}$ & $165 \mathrm{~W}$ & $\$ 18.22$ \\
Cree, Wolfspeed & SiC FET & $1.2 \mathrm{kV} / 31.6 \mathrm{~A}$ & $208 \mathrm{~W}$ & $\$ 16.67$ \\
\hline
\end{tabular}

From Table III, for voltages lesser than $1 \mathrm{kV}$ Si MOSFETs have relatively attractive power dissipation and costs. But at voltages higher than $1 \mathrm{kV}$, SiC FETs have considerably higher power dissipation than Si IGBT. The cost however is a major concern for SiC systems. As seen from Table II, Si IGBT has approximately 3 times lesser cost than its SiC counterpart. Also, a variety of Si IGBTs are available with better power dissipation characteristics at a comparable cost. SiC FETs show better switching characteristics than Si IGBTs and hence remain in competition despite its higher costs. Thus, the competition in this high voltage range is still neck and neck wherein both technologies have to achieve different milestones. Si IGBTs have to offer better switching characteristics without significant costs. SiC FETs must decrease their costs by better manufacturing and design techniques.

The battery charging circuit is a pretty straightforward circuit and utilizes both DC boost and DC buck conversion. Buck conversion is the inverse concept of the boost converter wherein it converts a high DC voltage level to a low voltage which is suitable for 
the battery charging. Recently, DC Fast charging of batteries is the emerging need with the rise of Electric Vehicles. Further clarification on the DC Fast Charging mechanism and the role of power transistors in it will be covered in the following section.

\section{Role of Power Transistors in the Electrification of Transportation Sector}

Recently there has been a major initiative towards electrification of surface transport by many countries. The aim of the initiative is to reduce global $\mathrm{CO}_{2}$ emissions to tackle the effects of climate change. It is not feasible to sustain the fossil fuel-based car industry anymore. Electrification of surface transport in the form of EVs is more efficient and reliable. As seen in (24), many countries have already adapted the integration of EVs into their transport sector. The growth of EV sales across the globe is given in reference (25).With the growth of the EV industry, there is a substantial increase in the demand for EV related components as well. We have already seen the growth of Li-iom batteries due to the increase in its demand by the transport sector in our earlier publication (5). Power transistors have also faced a similar incraese in demand due to their switching ability. As seen in the previous section, power transistors are crucial in DC-DC conversion and DC$\mathrm{AC}$ inversion. The significance of power transistors in the EV industry is quantified in two sectors: the internal DC-DC and DC-AC conversion mechanisms in the EV and the DC Fast Charging mechanisms.

\section{$\underline{\text { EV Internal Conversions }}$}

Apart from charging mechanisms, DC-DC conversion and DC-AC inversion is necessary in an EV internally. A typical block diagram for an EV can be seen in Fig. 6 (26). The DC-DC converter is utilized as a typical car battery like the internal combustion engine (ICE) automobiles for powering up many of the cars low-power requirements using the alternator. However, more than the DC-DC conversion, the focus in EVs is on the DC-AC conversion mechanism. The EV utilizes high-voltage batteries and converts the high DC voltage to an $\mathrm{AC}$ voltage which in turn drives the $\mathrm{AC}$ motors. The implementation of $\mathrm{AC}$ motors is an efficiency discussion which does not concern the scope of this paper.

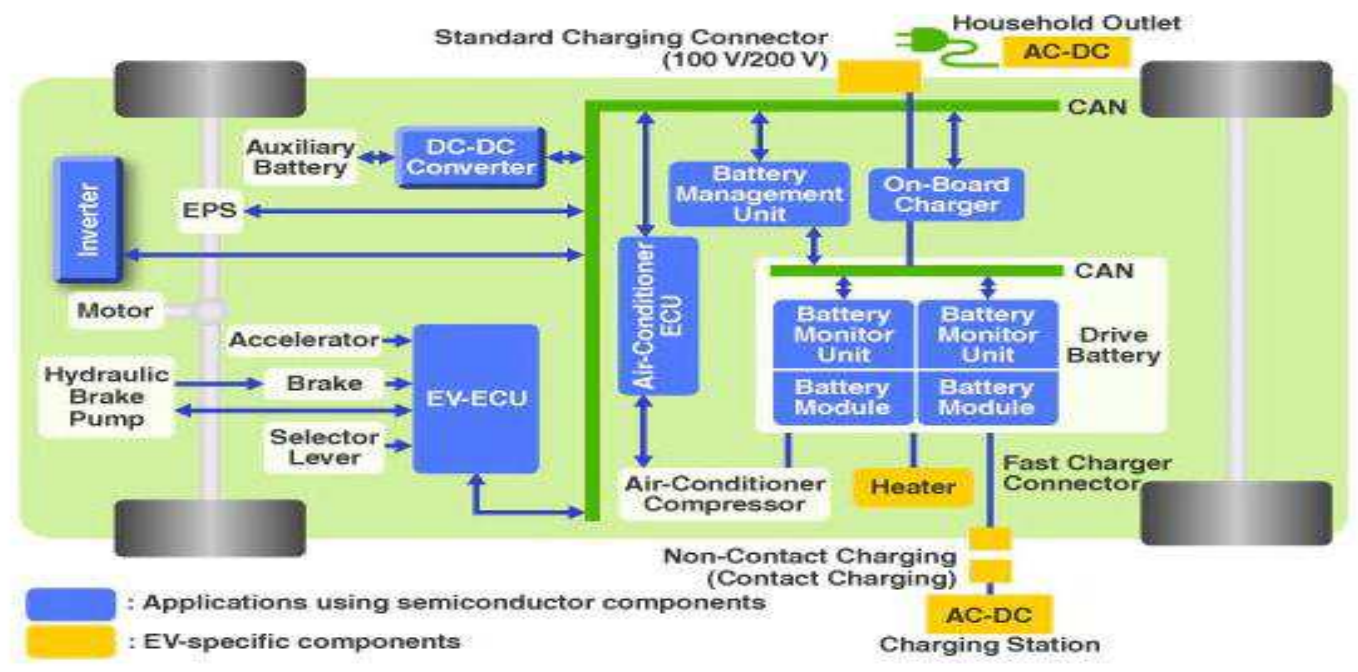

Figure 6. EV block diagram (26) 
Thus, in addition to efficient high-voltage inversion the mechanical reliability also need to be considered. In the previous section, Si SJ MOSFETs, Si IGBTs and SiC FETs displayed characterisitics to address the high-voltage operation. The real competition for high voltage performance above $1 \mathrm{kV}$ is between SiC FETs and Si IGBTs. Although SiC FETs have better switching characteristics and temperature performance than Si IGBTs at high voltages, cost is a concern for SiC FETs. The manufacturing cost is one factor that attributes for the cost of SiC FETs. Ultra large scale manufacturing and utilization of larger wafer size can bring down the cost of these transistors. Si manufacturers have already established their $300 \mathrm{~mm}$ wafer manufacturing ability. SiC manufacturers are however still moving from $150 \mathrm{~mm}$ wafers to $200 \mathrm{~mm}$ wafers (27). This attributes a disadvanatge for $\mathrm{SiC}$ technology in defect control, improving performance and yield. Thus, even though $\mathrm{SiC}$ FETs overpower Si IGBT in power density and switching efficiency, they still have to come a long way to match the cost advantage for Si IGBTs.

\section{Fast Charging for EV}

The current EV charging from the AC grid is based on inverting the AC voltage to $\mathrm{DC}$ and charging batteries using the DC-DC buck or boost converters. Fast charging technique on the other hand charge the batteries using a high voltage and current DC mechanisms to charge the battery at a much faster rate. Fig. 10 (28) illustrates the different charging times based on different charging techniques. The DC Fast charger employs high voltage operation and hence requires inverters and DC-DC boost converters for fast charging.

\section{Types of Electric Vehicle Charging Stations}
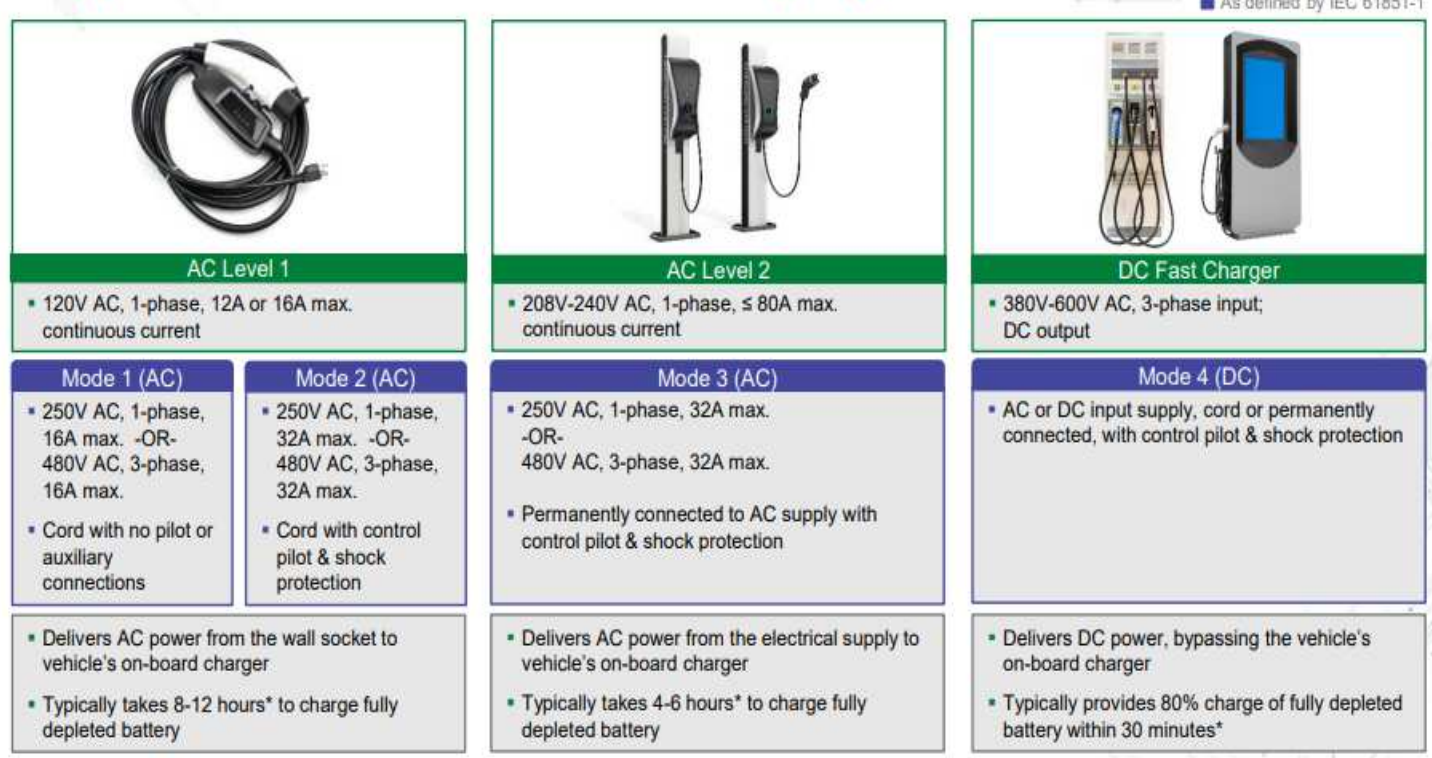

Figure 7. Types of EV charging techniques (28)

Since the future of EVs is concerned with their charging times, the DC fast charger is undergoing a lot of research and innovations. As with EV drive train, these fast chargers also implement high voltage FETs for the inversion and DC-DC boost conversion. Thus, SiC FETs and Si IGBTs have to battle for their place in this growing market. The argument for the transistors stays the same as the previous section. 


\section{Importance of DC Appliances}

As discussed in our recent paper (5), the new power network must be decentralized and based on local DC power networks for maximum efficiency and reliability. Most appliances that are in use today, except a few, are DC powered. Thus, if a DC power network is to be implemented, the utilization of DC appliances is beneficial with an energy savings point of view. Power losses are incurred when converting from AC to DC for the appliances and eliminating this conversion can improve the energy efficiency. In lieu of this argument, our project is to develop a DC powered induction cooktop for maximum power efficiency. The cooktop is catered towards eliminating the need for $\mathrm{CO}_{2}$ emitting cooking practices still prevalent in many rural parts of the world. The cooktop should utilize a PV and battery powered supply in providing a minimum cost technique for cooking. The role of transistors in such a cooktop is to provide the DC-DC boost conversion from the PV input and to invert the boosted DC voltage level to an AC voltage of suitable frequency for induction at highest efficiency. A comparison of the performance of different transistors like Si Power MOSFETs, Si SJ MOSFETs, GaN FETs and SiC FETs for the switching mechanism will be drawn out to decide the most cost-effective transistor for implementing the cooktop.

\section{Conclusion}

Ultimately, the current competition between transistors can be divided into two classes. Since Si Power MOSFETs provide the most cost-effective switching solution for low power applications, GaN transistors must compete with Si SJ MOSFETs to establish their viability in the $400-650 \mathrm{~V}$ range. For higher voltage operations of greater than $650 \mathrm{~V}, \mathrm{Si}$ IGBTs and $\mathrm{SiC}$ transistors are the competing technologies. However, newer Si SJ MOSFETs with high voltage abilities are being introduced to the market at attractive costs. The performance of these transistors on larger scale are yet to estimated. In the very high voltage range of greater than $1 \mathrm{kV}, \mathrm{SiC}$ transistors need to compete with $\mathrm{Si}$ IGBTs. Superior characteristics of $\mathrm{SiC}$ transistors coupled with decreased cost-to-market will prove to be the best technology for this scale of operation. As of today, $\mathrm{SiC}$ does not seem to come close to the cost for Si IGBTs. Thus, WBG transistors ( $\mathrm{GaN}$ and $\mathrm{SiC}$ ) need to improve on the manufacturing and design considerations to establish their market supremacy over $\mathrm{Si}$ technologies.

\section{References}

1. A. Asif and R. Singh, Further Cost Reduction of Battery Manufacturing. Batteries (2017)

2. Yole Developpement, Market\& Technology trends in Wide BandGap power packaging, APEC (2015).

3. F. Shah, H. Xiao, R. Li, M. Awais, G. Zhou and G. Bitew, Comparative performance evaluation of temperature dependent characteristics and power converter using GaN, SiC and Si power devices, in 2018 IEEE12th International Conference on Compatibility, Power Electronics and Power Engineering, pp. 1-7, IEEE (2018).

4. Forecast International's Energy Portal, Powerweb, Available at: http://www.fipowerweb.com/Renewable-Energy.html 
5. P. Paniyil, R. Singh, A. Asif, V. Powar, G. Bedi and J. Kimsey, Transformative and Disruptive Role of Local Direct Current Power Networks in Power and Transportation Sectors, Facta Universitatis, Series: Electronics and Energetics, Vol.32, pp. 387-402 (2019).

6. Inverter for the Solar Panel using an MC56F8023, Designer Reference Manual, Freescale Semiconductor (2011)

7. N. Baharudin, T. Mansur, F. Hamid, R. Ali, M. Misrun, Topologies of DC-DC Converter in Solar PV Applications, Indonesian Journal of Electrical Engineering and Computer Science, Vol. 8, No. 2, pp. 368-374 (2017).

8. K. Gopalakrishna, Frequency Characterizations of Si, SiC and GaN MOSFETs using Buck Converter in CCM as an Application, Master's Thesis, Wright State University (2013).

9. M. Slovick, Use SiC and GaN Power Components to Address EV Design Requirements, Digikey Electronics Article Library (2015).

10. N Sridhar, Demystifying high-voltage power electronics for solar inverters, Texas Instruments (2018).

11. Available at: https://www.mouser.com/

12. Fairchild (ON Semiconductor) FCPF190N65FL1 N-Channel SuperFET II FRFET MOSFET, $650 \mathrm{~V}, 20.6 \mathrm{~A}, 190 \mathrm{~m} \Omega$ Datasheet.

13. Vishay Siliconix, SiHA22N60AE, E Series Power MOSFET Datasheet.

14. STF26N60M2, STFI26N60M2, N-channel, $600 \mathrm{~V}, 0.14 \Omega$ typ., 20 A MDmesh M2, Power MOSFETs in TO-220FP and I PAKFP packages Datasheet.

15. Transphorm TPH3208PS, 650 V GaN FET TO-220 Series Datasheet.

16. ROHM Semiconductor, SCT3120ALHR, Automotive Grade N-channel SiC power MOSFET Datasheet.

17. Infineon, IPL65R099C7, MOSFET $650 \mathrm{~V}$ CoolMOS C7 Power Transistor Datasheet.

18. STW20N90K5, N-channel $900 \mathrm{~V}, 0.21 \Omega$ typ., 20 A MDmesh K5 Power MOSFET in a TO-247 package Datasheet.

19. Faichild (ON Semiconductor), FCP220N80, N-Channel SuperFET II MOSFET, $800 \mathrm{~V}, 23 \mathrm{~A}, 220 \mathrm{~m} \Omega$ Datasheet.

20. CREE, C3M0120090D, Silicon Carbide Power MOSFET, C3M MOSFET Technology, N-Channel Enhancement Mode Datasheet.

21. International Rectifier, IRG4PH30KDPbF, Insulated Gate Bipolar Transistor with Ultrafast Soft Recovery Diode Datasheet.

22. ROHM Semiconductor, SCT3080KL, N-channel SiC power MOSFET Datasheet.

23. CREE C2M0080120D, Silicon Carbide Power MOSFET, C2M MOSFET Technology, N-Channel Enhancement Mode Datasheet.

24. Countries leading the transition to Electric vehicles, Smart Energy International (2019).

25. Global EV Outlook 2019: Scaling up the transition to electric mobility, IEA (2019).

26. HEV/EV System, Toshiba Electronic Devices \& Storage Corporation, AmericasUnited States.

27. M. Lapedus, 200mm Cools Off, But Not for Long, Semiconductor Engineering (2019).

28. EV Charging Infrastructure: Supercharged Solutions to Enhance Safety, Efficiency, and Reliability, Littlefuse (2019). 